\title{
Minimal and complete factorization for a class of paraunitary filter banks
}

\author{
A K SOMAN \\ SignaLabs Corp., A-10 Rajyog, Vazira, Bombay 400 091, India \\ email: aksoman.sig@axcess.net.in
}

\begin{abstract}
M$ channel maximally decimated filter banks have been used in the past to decompose signals into subbands. The theory of perfect-reconstruction filter banks has also been studied extensively. A class of filter banks that has received particular attention are the so-called paraunitary filter banks. These filter banks are attractive because there exist robust structures for implementing these systems, based on factorizations of their polyphase matrices. In this paper, we will impose two other conditions on the paraunitary filter bank, the linear phase property of the filters, and their pairwise mirror-image symmetry in the frequency domain. These two properties are useful in several applications, including image processing. In this paper we will propose a factorization for filter banks having all the three properties simultaneously, namely, paraunitariness, linear phase, and pairwise mirror-image symmetry. We will then prove that our factorization is minimal and complete. This means that the number of delays used is equal to the McMillan degree, and that all filter banks satisfying the three properties simultaneously can be factored in terms of our factorization. Our factorization therefore gives us a robust structure for implementing these systems. All the three properties are guaranteed to be satisfied inspite of multiplier quantization.

We are not aware of any other factorizations that are proved minimal and complete for any three properties simultaneously.
\end{abstract}

Keywords. Paraunitary filter banks; linear phase; factorizations.

\section{Introduction}

Digital filter banks have been used in the past to decompose a signal into frequency subbands (Crochiere \& Rabiner 1983; Mintzer 1985; Smith \& Barnwell 1984; Vaidyanathan 1990; Vaidyanathan 1993; Vetterli 1986). The signals in different subbands are then coded and transmitted. Such schemes are popular for encoding data from speech, high quality audio, and image signals. The process of decomposing the signal and eventually reconstructing it is done by the 'analysis-synthesis' filter bank system shown in figure 5.4.1 of Vaidyanathan (1993). In this scheme, the $H_{i}(z)$ are the analysis filters and $F_{i}(z)$ are the synthesis filters. The boxes with $\downarrow M$ denote the decimators, or the subsampling devices, 
whereas the boxes with $\uparrow M$ denote the expanders, which increase the sampling rate. Their definitions are as in Crochiere \& Rabiner (1983) and Vaidyanathan (1990).

Figure 5.5.3 of Vaidyanathan (1993) is a representation of the subband coding scheme in terms of the polyphase matrices. $\mathbf{E}(z)$ is the polyphase matrix corresponding to the analysis filters, and $\mathbf{R}(z)$ is the polyphase matrix corresponding to the synthesis filters. The decimators and expanders have been moved across the polyphase matrices using the noble identities (Vaidyanathan 1990). It has been shown that it is indeed possible to perfectly reconstruct the original signal using such analysis-synthesis systems. One way to ensure perfect reconstruction is to let $\mathbf{R}(z)=\mathbf{E}^{-1}(z)$, and such a general scheme is called a biorthonormal system. Another approach to design a perfect reconstruction system with finite impulse response filters (FIR) is to choose the matrix $\mathbf{E}(z)$ to be a FIR 'paraunitary' matrix. A matrix is said to be paraunitary (Vaidyanathan 1993) if it satisfies the equation

$$
\widetilde{\mathbf{E}}(z) \mathbf{E}(z)=\mathbf{I},
$$

where $\widetilde{\mathbf{E}}(z)=\mathbf{E}^{\dagger}\left(1 / z^{*}\right)$. The system can be guaranteed to have the perfect reconstruction property by having $\mathbf{R}(z)=\widetilde{\mathbf{E}}(z)$.

Consider the synthesis bank of figure 1 of Vaidyanathan (1993). The original signal can be written in terms of the subband signals as

$$
x(n)=\sum_{k=0}^{M-1} \sum_{m} y_{k}(m) f_{k}(n-M m) .
$$

This can be viewed as a representation of the original signal in terms of a doubly indexed set of basis functions $\eta_{k m}(n)=f_{k}(n-M m)$. It is known (Rioul \& Vetterli 1991; Soman \& Vaidyanathan 1992) that this set of basis functions is orthonormal if and only if the polyphase matrix $\mathbf{R}(z)$ corresponding to these filters is paraunitary.

Another feature of the paraunitary analysis-synthesis system is that the analysis and synthesis filters are simply time-reversed conjugate versions of each other and, in particular therefore, they are of the same length. In a practical subband coding system, both the filter coefficients, as well as the subband signals are quantized. It has been shown (Vaidyanathan 1993) that there exist structures which retain the paraunitary property inspite of coefficient quantization. The perfect-reconstruction property is however lost, when the signals in each subband are quantized. A paraunitary system still has some important features in the presence of subband quantization, which are listed in Soman \& Vaidyanathan (1993).

In several applications, and particularly in image coding, it is desirable to have each filter in the system as a linear phase filter. This would not be necessary if there were no subband quantization, which is not a case of practical interest. The problem of designing linear phase perfect reconstruction systems has been considered by other authors in the past (Princen \& Bradley 1986; Vetterli \& Le Gall 1989). Nguyen \& Vaidyanathan (1988) have studied the condition that the analysis (and synthesis) filters satisfy the pairwise mirror-image symmetry constraint in the frequency domain around $\pi / 2$. The advantage of the resulting structure is that it requires fewer parameters. The design time is correspondingly lower than other structures. The filter responses obtained using this structure are also better and have been widely used.

A natural question which arises is whether all these three properties can be simultaneously imposed on a filter bank, i.e., is it possible to have paraunitary filter banks whose filters have linear phase, and also satisfy the pairwise mirror-image condition in the frequency domain? Such a filter bank, if it exists, would have all the desirable properties listed above. In this paper, we will show the existence of such filter banks. The method 
we use is that of factorizing the polyphase matrices. Our factorizations are complete. This means that all filter banks having these properties can be factorized in terms of our structure. Hence, the implementations based on our factorization are robust to quantization. The three desirable properties continue to hold even if we quantize the multipliers in our structure.

Another point worth mentioning is the minimality of our structure. A structure is said to be minimal if it uses the minimum number of delay elements necessary (Vaidyanathan 1993). We will also show that our structure is minimal.

\subsection{Notations}

Bold-faced quantities denote matrices and vectors, as in $\mathbf{A}$ and $\mathbf{x} \cdot \mathbf{A}^{T}, \mathbf{A}^{-1}$ and $\operatorname{Tr}(\mathbf{A})$ denote the transpose, the inverse, and the trace of the matrix $\mathbf{A}$ respectively. A subscript on a matrix indicates its size, when the size is not clear from the context. Reserved symbols for special matrices are as follows: $\mathbf{I}$ is the identity matrix. The matrix $\mathbf{J}_{N}$ is the anti-diagonal matrix of size $N \times N$. For example, the anti-diagonal matrix of size 4 is

$$
\mathbf{J}_{4}=\left[\begin{array}{llll}
0 & 0 & 0 & 1 \\
0 & 0 & 1 & 0 \\
0 & 1 & 0 & 0 \\
1 & 0 & 0 & 0
\end{array}\right] .
$$

$\mathbf{0}$ will denote the null matrix, whose size will be clear from the context. $\mathbf{V}_{M}$ will denote a special diagonal matrix of size $M \times M$, with alternating $\pm 1^{\prime} s$ on the diagonal, starting with +1. Hence if $M / 2$ is even, we can write $\mathbf{V}_{M}=\left(\begin{array}{cc}\mathbf{V}_{M / 2} & 0 \\ 0 & \mathbf{V}_{M / 2}\end{array}\right)$, whereas if $M / 2$ is odd, $\mathbf{V}_{M}=\left(\begin{array}{cc}\mathbf{V}_{M / 2} & \mathbf{0} \\ \mathbf{0} & -\mathbf{V}_{M / 2}\end{array}\right)$. The matrix $\mathbf{Q}$ is by definition, $\mathbf{Q}=\left(\begin{array}{cc}\mathbf{V}_{M / 2} & \mathbf{0} \\ \mathbf{0} & -\mathbf{V}_{M / 2}\end{array}\right)$ for even $M / 2$, A superscript asterisk as in $f^{*}(n)$ denotes conjugation. Consider a transfer function $A(z)$. It can be written in terms of its $M$ polyphase components (Vaidyanathan 1990) as follows:

$$
A(z)=a_{0}\left(z^{M}\right)+z^{-1} a_{1}\left(z^{M}\right)+\ldots+z^{-(M-1)} a_{M-1}\left(z^{M}\right) .
$$

This is known as Type I polyphase. Let $H_{i}(z), i=0, \ldots, M-1$, be a set of analysis filters. They can be written as

$$
H_{k}(z)=\sum_{l=0}^{M-1} z^{-l} E_{k l}\left(z^{M}\right) \quad k=0, \ldots, M-1 .
$$

The matrix $\mathbf{E}(z)=\left[E_{k, l}(z)\right]$ is called the polyphase matrix of the analysis filters. A set of filters $H_{k}(z)$ whose polyphase matrix is paraunitary are said to form a paraunitary system (1). Throughout this paper, we will deal with real, causal, FIR systems. Given such a system $\mathbf{E}(z)$ of order $N$, we can write it explicitly as

$$
\mathbf{E}(z)=\mathbf{e}(0)+\mathbf{e}(1) z^{-1}+\mathbf{e}(2) z^{-2}+\ldots+\mathbf{e}(N) z^{-N}, \quad \mathbf{e}(N) \neq \mathbf{0} .
$$

In this case, the analysis filters typically have order $M(N+1)-1$.

\section{Linear phase paraunitary filters with pairwise mirror-image frequency responses}

We will now propose our factorization for linear-phase paraunitary matrices with pairwise mirror-image symmetry. We will deal with the case of $M$ being even, which is the case 
almost always used in practice. In order to obtain factorizations of such systems, we first need to obtain a characterization of their polyphase matrix which reflects these properties of the individual filters. The polyphase matrix of a paraunitary system can be characterised as in (1). Now, consider a set of $M$ paraunitary transfer functions whose polyphase matrix E $(z)$ satisfies the property (Vetterli \& Le Gall 1989)

$$
\mathbf{D} z^{-N} \mathbf{E}\left(z^{-1}\right) \mathbf{J}_{M}=\mathbf{E}(z)
$$

where $N$ is the order of the paraunitary matrix $\mathbf{E}(z)$. Such a polyphase matrix corresponds to a set of filters which have linear phase. The matrix $\mathbf{D}$ is a diagonal matrix whose entries are $\pm 1^{\prime} s$, the $+1^{\prime} s$ in those rows which correspond to symmetric filters and -1 's in those that correspond to antisymmetric filters. The filters described by this equation have the same centre of symmetry $((N+1) M-1) / 2$.

The condition that the filters in the filter-bank satisfy the pairwise mirror-image symmetry often leads to better filter designs, and faster convergence of the optimization process. An example of a structure satisfying these properties was given by Soman et al (1993). Consider figure 3 of that paper. The filters at the $m$ th stage are denoted as $H_{m, i}(z)$. If these filters satisfy pairwise mirror-image symmetry, they can be related to each other as

$$
H_{m, M-1-k}(z)=z^{-((m+1) M-1)} H_{m, k}\left(-z^{-1}\right), \quad k=0, \ldots, M-1 .
$$

The order of each filter is $(m+1) M-1$. It can be verified that in this case, the polyphase matrix $\mathbf{E}_{m}(z)$ of the filters satisfies the matrix equation

$$
z^{-m} \mathbf{Y} \mathbf{E}_{m}\left(z^{-1}\right) \mathbf{V}_{M} \mathbf{J}_{M}=\mathbf{E}_{m}(z)
$$

where

$$
\mathbf{Y}=\left(\begin{array}{cc}
\mathbf{0} & -\mathbf{J}_{M / 2} \\
\mathbf{J}_{M / 2} & \mathbf{0}
\end{array}\right)
$$

Under the linear phase condition mentioned earlier, the equation can be further simplified to (assuming $M$ is even)

$$
\mathbf{J}_{M} \mathbf{E}(z)=\mathbf{E}(z) \mathbf{V}_{M}
$$

Theorem. A linear phase paraunitary matrix satisfying (6) whose filters satisfy the additional painwise mirror-image property in the frequency domain, (10), can always be realized as

$$
\mathbf{E}(z)=\mathbf{S} \Lambda(z) \mathbf{P} \mathbf{T}_{0} \Lambda(z) \ldots \mathbf{T}_{N} \mathbf{P}
$$

where

$$
\begin{aligned}
& \mathbf{S}=(1 / \sqrt{ } 2)\left(\begin{array}{cc}
\mathbf{S}_{0} & \mathbf{0} \\
\mathbf{0} & \mathbf{J}_{M / 2} \mathbf{S}_{0}
\end{array}\right)\left(\begin{array}{cc}
\mathbf{I} & \mathbf{J}_{M / 2} \\
\mathbf{I} & -\mathbf{J}_{M / 2}
\end{array}\right) \mathbf{Q} \\
& \mathbf{T}_{i}=\left(\begin{array}{cc}
\mathbf{I}_{M / 2} & \mathbf{I}_{M / 2} \\
\mathbf{I}_{M / 2} & -\mathbf{I}_{M / 2}
\end{array}\right)\left(\begin{array}{cc}
\mathbf{V}_{M / 2} \mathbf{U}_{i} \mathbf{V}_{M / 2} & \mathbf{0} \\
\mathbf{0} & \mathbf{U}_{i}
\end{array}\right)\left(\begin{array}{cc}
\mathbf{I}_{M / 2} & \mathbf{I}_{M / 2} \\
\mathbf{I}_{M / 2} & -\mathbf{I}_{M / 2}
\end{array}\right) \\
& \Lambda(z)=\left(\begin{array}{cc}
\mathbf{I}_{M / 2} & \mathbf{0} \\
\mathbf{0} & z^{-1} \mathbf{I}_{M / 2}
\end{array}\right)
\end{aligned}
$$

and 


$$
\mathbf{P}=\left(\begin{array}{cc}
\mathbf{I}_{M / 2} & \mathbf{0} \\
\mathbf{0} & \mathbf{J}_{M / 2}
\end{array}\right)
$$

$\mathrm{S}_{0}$ and $\mathrm{U}_{i}$ are arbitrary orthogonal matrices, and $\mathbf{Q}$ is a symmetric permutation. $\diamond \diamond \diamond$

To develop a cascade structure which generates such filters, we will assume that we have a paraunitary matrix $\mathbf{E}_{m-1}(z)$ of order $m-1$ satisfying the conditions of paraunitariness (1), linear phase (6), and pairwise mirror-image symmetry of frequency responses (10). From it, we will show how a paraunitary matrix $\mathbf{E}_{m}(z)$ of order $m$ can be obtained satisfying the above three properties. We will do this by post multiplying the given matrix $\mathbf{E}_{m-1}(z)$ by a paraunitary matrix $\mathbf{R}(z)$ of order one.

Let

$$
\mathbf{E}_{m}(z)=\mathbf{E}_{m-1}(z) \mathbf{R}(z) .
$$

Clearly, $\mathbf{E}_{m}(z)$ is paraunitary if $\mathbf{E}_{m-1}(z)$ and $\mathbf{R}(z)$ are both paraunitary. Also,

$$
\mathbf{E}_{m-1}(z)=\mathbf{E}_{m}(z) \tilde{\mathbf{R}}(z) .
$$

Propagating the linear phase property: From the fact that $\mathbf{E}_{m-1}(z)$ satisfies the linear phase property, and substituting from (17), we have

$$
z^{-(m-1)} \mathbf{D} \mathbf{E}_{m}\left(z^{-1}\right) \widetilde{\mathbf{R}}\left(z^{-1}\right) \mathbf{J}_{M}=\mathbf{E}_{m}(z) \widetilde{\mathbf{R}}(z),
$$

i.e.,

$$
z^{-(m-1)} \mathbf{D} \mathbf{E}_{m}\left(z^{-1}\right) \tilde{\mathbf{R}}\left(z^{-1}\right) \mathbf{J}_{M} \mathbf{R}(z)=\mathbf{E}_{m}(z) .
$$

Hence for $\mathbf{E}_{m}(z)$ to satisfy the linear phase property, $\mathbf{R}(z)$ should satisfy

$$
\widetilde{\mathbf{R}}\left(z^{-1}\right) \mathbf{J}_{M} \mathbf{R}(z)=z^{-1} \mathbf{J}_{M} \text {. }
$$

It can be verified that this equation is satisfied if $\mathbf{R}(z)=\Lambda(z) \mathbf{P T} \mathbf{T}_{m} \mathbf{P}$ with the matrices $\Lambda(z), \mathbf{P}$ and $\mathbf{T}$ as stated in the theorem.

Propagating the pairwise mirror-image property in the frequency domain: Assuming that (10) holds for $\mathbf{E}_{m-1}(z)$, and using (17) we get,

$$
\mathbf{J}_{M} \mathbf{E}_{m}(z) \widetilde{\mathbf{R}}(z)=\mathbf{E}_{m}(z) \widetilde{\mathbf{R}}(z) \mathbf{V}_{M},
$$

i.e.,

$$
\mathbf{J}_{M} \mathbf{E}_{m}(z)=\mathbf{E}_{m}(z) \widetilde{\mathbf{R}}(z) \mathbf{V}_{M} \mathbf{R}(z) .
$$

Hence, $\mathbf{R}(z)$ should satisfy the property

$$
\tilde{\mathbf{R}}(z) \mathbf{V}_{M} \mathbf{R}(z)=\mathbf{V}_{M}
$$

We now have two cases. Case 1: $M / 2$ is even, in which case, $\mathbf{V}_{M}=\left(\begin{array}{cc}\mathbf{V}_{M / 2} & 0 \\ 0 & \mathbf{V}_{M / 2}\end{array}\right)$, or Case 2: $M / 2$ is odd, in which case $V_{M}=\left(\begin{array}{cc}V_{M / 2} & 0 \\ 0 & -V_{M / 2}\end{array}\right)$. In either case, using the fact that $\mathbf{R}(z)=\Lambda(z)$ PTP with the various matrices as in the statement of the theorem, upon simplification we can verify that $(23)$ is indeed satisfied. Thus all three properties; paraunitariness, linear phase, and pairwise mirror-symmetry have been satisfied by the new matrix $\mathbf{E}_{m}(z)$.

Initialization: It only remains to find a degree zero paraunitary matrix $\mathbf{E}_{0}(z)$ (i.e. a constant orthogonal matrix $\mathbf{S}$ ), which will initialize the above process. It can be verified that the matrix $\mathbf{S}$ satisfies the linear phase property $\left(\mathbf{D S J} \mathbf{J}_{M}=\mathbf{S}\right)$, if it is of the form 


$$
\mathbf{S}=(1 / \sqrt{ } 2)\left(\begin{array}{cc}
\mathbf{S}_{0} & \mathbf{0} \\
\mathbf{0} & \mathbf{S}_{1}
\end{array}\right)\left(\begin{array}{cc}
\mathbf{I}_{M / 2} & \mathbf{J}_{M / 2} \\
\mathbf{I}_{M / 2} & -\mathbf{J}_{M / 2}
\end{array}\right) \mathbf{Q}
$$

where $\mathbf{Q}$ is a symmetric permutation matrix. This is because $\mathbf{Q} \mathbf{J}_{M} \mathbf{Q}=\mathbf{J}_{M}$ for any such permutation matrix. Let $\mathbf{Q}$ be so chosen that $\mathbf{Q} \mathbf{V}_{M} \mathbf{Q}=\mathbf{D}$, where $\mathbf{D}=\left(\begin{array}{cc}\mathbf{I}_{M / 2} & 0 \\ \mathbf{0} & -\mathbf{I}_{M / 2}\end{array}\right)$. Now, let $\mathbf{S}^{\prime}=\mathbf{S Q}$. For the matrix $\mathbf{S}$ to satisfy the pairwise mirror-image property $\left(\mathbf{J}_{M} \mathbf{S}=\right.$ $\mathbf{S V}_{M}$ ), it can be verified that the matrix $\mathbf{S}^{\prime}$ should satisfy $\mathbf{S}^{\prime} \mathbf{D \mathbf { S } ^ { \prime }}=\mathbf{J}_{M}$. Substituting the forms of various matrices and simplifying, we get

$$
\left(\begin{array}{cc}
\mathbf{0} & \mathbf{S}_{0} \mathbf{S}_{1}^{T} \\
\mathbf{S}_{1} \mathbf{S}_{0}^{T} & \mathbf{0}
\end{array}\right)=\left(\begin{array}{cc}
\mathbf{0} & \mathbf{J}_{M / 2} \\
\mathbf{J}_{M / 2} & \mathbf{0}
\end{array}\right)
$$

This equation can be satisfied by letting $\mathbf{S}_{0}$ be an arbitrary orthogonal matrix, and choosing $\mathbf{S}_{1}=\mathbf{J}_{M / 2} \mathbf{S}_{0}$. Thus the matrix $\mathbf{S}$ can be realized with $\left(\begin{array}{c}M / 2 \\ 2\end{array}\right)$ rotations (Murnaghan 1962).

Proof of completeness. In this section, we will start with a paraunitary matrix $\mathbf{E}_{m}(z)$ of order $m$ satisfying the conditions of paraunitariness (1), linear phase (6), and pairwise mirror-image symmetry of frequency responses (10). From it, we will show how the matrix $\mathbf{E}_{m-1}(z)$ of order $m-1$ as in (17) satisfies all three of the said properties. For brevity, we will only talk about the case $M / 2=e v e n$, since the proofs for $M / 2=o d d$ are identical, except for bookkeeping.

The paraunitariness of $\mathbf{E}_{m-1}(z)$ is obvious from (17) itself.

Linear phase property: From the fact that $\mathbf{E}_{m}(z)$ satisfies the linear phase property, we have

$$
z^{-m} \mathbf{D E} \mathbf{E}_{m}\left(z^{-1}\right) \mathbf{J}_{M}=\mathbf{E}_{m}(z)
$$

i.e.,

$$
z^{-m} \mathbf{D} \mathbf{E}_{m-1}\left(z^{-1}\right) \mathbf{R}\left(z^{-1}\right) \mathbf{J}_{M} \widetilde{\mathbf{R}}(z)=\mathbf{E}_{m-1}(z) .
$$

Noting the form of the matrix $\mathbf{R}(z)=\Lambda(z) \mathbf{P T} \mathbf{T}_{m} \mathbf{P}$, and its component matrices as mentioned in the statement of the theorem, one can verify that the above simplifies to

$$
z^{-(m-1)} \mathbf{D E}_{m-1}\left(z^{-1}\right) \mathbf{J}_{M}=\mathbf{E}_{m-1}(z),
$$

implying that $\mathbf{E}_{m-1}(z)$ has linear phase.

Pairwise mirror-image property in the frequency domain: Assuming that (10) holds for $\mathbf{E}_{m}(z)$, and using (16) we get,

$$
\mathbf{J}_{M} \mathbf{E}_{m-1}(z) \mathbf{R}(z)=\mathbf{E}_{m-1}(z) \mathbf{R}(z) \mathbf{V}_{M},
$$

i.e.,

$$
\mathbf{J}_{M} \mathbf{E}_{m-1}(z)=\mathbf{E}_{m}(z) \mathbf{R}(z) \mathbf{V}_{M} \widetilde{\mathbf{R}}(z) .
$$

Again, from $\mathbf{R}(z)=\Lambda(z) \mathbf{P} \mathbf{T}_{m} \mathbf{P}$, and its component matrices as in the statement of the theorem, it can be verified that

$$
\mathbf{R}(z) \mathbf{V}_{M} \widetilde{\mathbf{R}}(z)=\mathbf{V}_{M},
$$

for both cases $M / 2$ being odd/even.

Causality: We now have to show that there exists a matrix $\mathbf{R}(z)$ of the form $\mathbf{R}(z)=$ $\Lambda(z) \mathbf{P} \mathbf{T}_{m} \mathbf{P}$ such that $\mathbf{E}_{m-1}(z)$ obtained from (17) is causal. The linear phase property, 
the pairwise mirror-image property, and the paraunitary property continue to hold for the reduced system as long as the matrix $\mathbf{R}(z)$, is any orthogonal matrix of this required form. Indeed, it is the causality condition on the reduced system which determines the particular choice of the matrix $\mathbf{R}(z)$. Since $\mathbf{E}_{m-1}(z)=\mathbf{E}_{m}(z) \widetilde{\mathbf{R}}(z)$, and knowing the form of $\mathbf{R}(z)$, we get,

$$
\mathbf{E}_{m-1}(z)=\mathbf{E}_{m}(z) \mathbf{P T} \mathbf{T}_{m}^{T} \mathbf{P} \Lambda^{-1}(z)
$$

Noting the form of $\Lambda(z)$ from the statement of the theorem, we get

$$
\mathbf{E}_{m-1}(z)=\mathbf{E}_{m}(z) \mathbf{P} \mathbf{T}_{m}^{T} \mathbf{P}\left(\begin{array}{cc}
\mathbf{I}_{M / 2} & \mathbf{0} \\
\mathbf{0} & \mathbf{0}
\end{array}\right)+\mathbf{E}_{m}(z) \mathbf{P} \mathbf{T}_{m}^{T} \mathbf{P}\left(\begin{array}{cc}
\mathbf{0} & \mathbf{0} \\
\mathbf{0} & z \mathbf{I}_{M / 2}
\end{array}\right)
$$

The second term on the right hand side of this equation is responsible for the non-causality. In particular, the noncausal part of the second term is given by

$$
\mathbf{e}_{m}(0) \mathbf{P T} \mathbf{T}_{m}^{T} \mathbf{P}\left(\begin{array}{cc}
\mathbf{0} & \mathbf{0} \\
\mathbf{0} & z \mathbf{I}_{M / 2}
\end{array}\right)
$$

where we have used an expansion similar to (5) for $\mathbf{E}(z)$.

We have to show that there exists a matrix $\mathbf{T}_{m}$ of the form in (13) which makes this term equal to zero. Let us simplify this term which needs to be made zero. Substituting the form of the matrices $\mathbf{P}$ and $\mathbf{T}_{m}$ from (14) and (13) we have, the non-causal part

$$
\begin{aligned}
\mathbf{e}_{m}(0) & \mathbf{P T} T_{m}^{T} \mathbf{P}\left(\begin{array}{cc}
\mathbf{0} & \mathbf{0} \\
\mathbf{0} & \mathbf{I}_{M / 2}
\end{array}\right) \\
= & \mathbf{e}_{m}(0)\left(\begin{array}{cc}
\mathbf{0} & \left(\mathbf{V}_{M / 2} \mathbf{U}_{m}^{T} \mathbf{V}_{M / 2}+\mathbf{U}_{m}^{T}\right) \mathbf{J}_{M / 2} \\
\mathbf{0} & \mathbf{J}_{M / 2}\left(\mathbf{V}_{M / 2} \mathbf{U}_{m}^{T} \mathbf{V}_{M / 2}+\mathbf{U}_{m}^{T}\right) \mathbf{J}_{M / 2}
\end{array}\right) .
\end{aligned}
$$

Hence we need to show the existence of a orthogonal matrix $\mathbf{U}_{m}$ such that

$$
\mathbf{e}_{m}(0)\left(\begin{array}{c}
\left(\mathbf{V}_{M / 2} \mathbf{U}_{m}^{T} \mathbf{V}_{M / 2}+\mathbf{U}_{m}^{T}\right) \\
\mathbf{J}_{M / 2}\left(\mathbf{V}_{M / 2} \mathbf{U}_{m}^{T} \mathbf{V}_{M / 2}+\mathbf{U}_{m}^{T}\right)
\end{array}\right)=\mathbf{0}
$$

Notice that this matrix multiplying $\mathbf{e}_{m}(0)$ has $M$ rows, but $M / 2$ columns. We will now proceed to show that such a matrix $\mathbf{U}_{m}$ always exists. Firstly, let us examine the above equation closely. Given the nature of the matrix $\mathbf{V}_{M / 2}$, the matrix that multiplies $\mathbf{e}_{m}(0)$ in (36) is of the form

$$
\left(\begin{array}{c}
\left(\mathbf{V}_{M / 2} \mathbf{U}_{m}^{T} \mathbf{V}_{M / 2}+\mathbf{U}_{m}^{T}\right) \\
\mathbf{J}_{M / 2}\left(\mathbf{V}_{M / 2} \mathbf{U}_{m}^{T} \mathbf{V}_{M / 2}+\mathbf{U}_{m}^{T}\right)
\end{array}\right)=\left(\begin{array}{ccccccc}
X & 0 & X & 0 & \ldots & X & 0 \\
0 & X & 0 & X & \ldots & 0 & X \\
X & 0 & X & 0 & \ldots & X & 0 \\
0 & X & 0 & X & \ldots & 0 & X \\
\vdots & \vdots & \vdots & \vdots & \ddots & \vdots & \vdots \\
X & 0 & X & 0 & \ldots & X & 0 \\
0 & X & 0 & X & \ldots & 0 & X \\
X & 0 & X & 0 & \ldots & X & 0
\end{array}\right),
$$

where $X$ represent non-zero entries. The matrix thus has a checker-board pattern. Now, paraunitary condition in the time domain implies that $\mathbf{e}_{m}(0) \mathbf{e}_{m}^{T}(m)=\mathbf{0}$. Also, (6) in particular means that

$$
\operatorname{De}_{m}(0) \mathbf{J}_{M}=\mathbf{e}_{m}(m)
$$


Hence we have

$$
\mathbf{e}_{m}(0) \mathbf{J}_{M} \mathbf{e}_{m}^{T}(0)=\mathbf{0} .
$$

By Sylvester's rank inequality, the above means that $\operatorname{rank}\left(\mathbf{e}_{m}(0)\right)=r \leq M / 2$. On the other hand, (10) in particular implies that $\mathbf{J}_{M} \mathbf{e}_{m}(0)=\mathbf{e}_{m}(m) \mathbf{V}_{M}$. Combining this with the paraunitary condition we get

$$
\mathbf{e}_{m}(0) \mathbf{J}_{M} \mathbf{V}_{M} \mathbf{e}_{m}^{T}(0)=\mathbf{0} .
$$

Equations (36) and (37) tell us that we need to prove the existence of $M / 2$ checkered vectors that are orthogonal to the rows of $\mathbf{e}_{m}(0)$. Equation (39) implies that

$$
\mathbf{W} \mathbf{e}_{m}(0) \mathbf{J} \mathbf{e}_{m}^{T}(0) \mathbf{W}^{T}=\mathbf{0},
$$

for any matrix $\mathbf{W}$. For the same matrix $\mathbf{W},(40)$ implies that

$$
\mathbf{W e}_{m}(0) \mathbf{J V e} \mathbf{e}_{m}^{T}(0) \mathbf{W}^{T}=\mathbf{0} .
$$

Let the matrix $\mathbf{W}$ be so chosen that the first $r$ rows of the matrix $\mathbf{W e}_{m}(0)$ form an orthonormal basis of real vectors $\mathbf{x}_{i}$ for the rows of the matrix $\mathbf{e}_{m}(0)$. (This is possible, since the matrix $\mathbf{e}_{m}(0)$ is itself real, whose rank is $r$.) These vectors $\mathbf{x}_{i}$, being orthonormal, satisfy $\mathbf{x}_{i}^{T} \mathbf{x}_{j}=0, \quad(i \neq j)$. Equations (40) and (41) tell us that the same vectors also satisfy the conditions $\mathbf{x}_{i}^{T} \mathbf{J} \mathbf{x}_{j}=0$ and $\mathbf{x}_{i}^{T} \mathbf{J V \mathbf { x } _ { j }}=0$ for all $i, j$.

Each of these vectors $\mathbf{x}_{i}$ can be written in terms of its components $\mathbf{a}_{i}$ and $\mathbf{b}_{i}$, where these two vectors have the checkered form shown below

$$
\mathbf{a}_{i}^{T}=\left(\begin{array}{c}
X \\
0 \\
X \\
0 \\
X \\
0 \\
\vdots \\
X \\
0
\end{array}\right) \quad \text { and } \quad \mathbf{b}_{i}^{T}=\left(\begin{array}{c}
0 \\
X \\
0 \\
X \\
0 \\
X \\
\vdots \\
0 \\
X
\end{array}\right)
$$

Hence the vectors $\mathbf{a}_{i}$ and $\mathbf{b}_{i}$ have alternating entries equal to zero, with the entries that are zero in $\mathbf{a}_{i}$ being non-zero in $\mathbf{b}_{i}$, and vice-versa. Moreover, since $\mathbf{x}_{i}$ are real, so are $\mathbf{a}_{i}$ and $b_{i}$. Then, from (41) we have

$$
\left(\mathbf{a}_{i}+\mathbf{b}_{i}\right) \mathbf{J}\left(\mathbf{a}_{j}+\mathbf{b}_{j}\right)^{T}=0,
$$

and since $M$ is even, this simplifies to

$$
\mathbf{a}_{i} \mathbf{J b}_{j}^{T}+\mathbf{b}_{i} \mathbf{J a}_{j}^{T}=0 .
$$

From (42) we have

$$
\left(\mathbf{a}_{i}+\mathbf{b}_{i}\right) \mathbf{J V}\left(\mathbf{a}_{j}+\mathbf{b}_{j}\right)^{T}=0 .
$$

But for even $M, \mathbf{V a}_{i}^{T}=\mathbf{a}_{i}^{T}$, and $\mathbf{V b} \mathbf{b}_{i}^{T}=-\mathbf{b}_{i}^{T}$, and hence the above equation becomes

$$
-\mathbf{a}_{i} \mathbf{J b}_{j}^{T}+\mathbf{b}_{i} \mathbf{J a}_{j}^{T}=0 .
$$


Using (45) and (47), we get $\mathbf{a}_{i} \mathbf{J b}_{j}^{T}=0$, for all $i, j$. This means that the rows of the matrix $\mathbf{e}_{m}(0)$ are orthogonal to any linear combination of $\mathbf{a}_{i}$, and any linear combination of $\mathbf{b}_{i}$ for $i=1, \ldots, r$. Now choose $r$ linear combinations of $\mathbf{a}_{i}$ which are mutually orthogonal, and $r$ linear combinations of $\mathbf{b}_{i}$ that are mutually orthogonal. Hence you have $2 r$ orthogonal vectors. Each of them is checkered (since $\mathbf{a}_{i}$ and $\mathbf{b}_{i}$ are checkered). Denote by $\varepsilon_{1}$ the subspace spanned by all the vectors that are checkered and whose odd entries are zeros (for example, the vectors $\mathbf{a}_{i}$ lie in $\varepsilon_{1}$ ). In this subspace $\varepsilon_{1}$ there are $(M / 2)-r$ vectors which are orthogonal to these $r$ vectors $\mathbf{a}_{i}$, since the dimension of the space $\varepsilon_{1}$ is $M / 2$. Similarly denote by $\varepsilon_{2}$ the subspace spanned by all the vectors that are checkered and whose even entries are zeros (for example, the vectors $\mathbf{b}_{i}$ lie in $\varepsilon_{2}$ ). In the subspace $\varepsilon_{2}$ there are $(M / 2)-r$ vectors which are orthogonal to these $r$ vectors $\mathbf{b}_{i}$, since the dimension of the space $\varepsilon_{2}$ is $M / 2$. Using these two sets of vectors along with the original $2 r$ vectors give us a orthogonal basis of $M / 2$ checkered vectors, such that they are orthogonal to the rows of the matrix $\mathbf{e}_{m}(0)$. It is clear that the matrix with these $M / 2$ vectors as its columns is a checkered matrix of the required form in (37). (It is also easily verified that the matrix obtained by flipping the rows of the bottom half, and then subtracting the bottom half from the top half of this matrix gives rise to an $M / 2$ by $M / 2$ orthogonal matrix, which we will call $\mathbf{U}_{m}^{T}$ in (36). Hence we have shown that there always exists a matrix $\mathbf{T}_{m}$ of the form mentioned in the statement of the theorem, such that the reduced system is causal.

Order reduction: Given the fact that $\mathbf{E}_{m}(z)$ is causal, and that it satisfies (6), we can see that the order of $\mathbf{E}_{m}(z)$ is $m$. Thus there is a reduction in order by 1 . Hence for a system of order $N$, the factorization process is guaranteed to terminate in $N$ steps.

This concludes the proof of the theorem. $\diamond \diamond \diamond$

In the above theorem, each orthogonal building block can be realized with $\left(\begin{array}{c}M / 2 \\ 2\end{array}\right)$ angles (variables).

The degree of a causal rational system is defined as (Vaidyanathan 1993) the minimum number of delays required for its implementation. A structure is said to be minimal if the number of delays used is equal to the degree of the transfer function. For a paraunitary system, we know that (theorem 14.7.1 of Vaidyanathan (1993))

$$
\operatorname{deg}[\operatorname{det}[\mathbf{E}(z)]]=\operatorname{deg}[\mathbf{E}(z)] \text {. }
$$

In our case,

$$
\operatorname{deg}[\operatorname{det}[\mathbf{E}(z)]]=\operatorname{deg}\left[\operatorname{det}\left[\mathbf{S} \Lambda(z) \mathbf{P} \mathbf{T}_{0} \Lambda(z) \cdots \cdots \Lambda(z) \mathbf{T}_{N} \mathbf{P}\right]\right]=N M / 2,
$$

which is equal to the number of delays used. Hence our factorization is minimal.

\section{Conclusions}

In this paper, we proposed and proved a factorization for linear-phase paraunitary filter banks whose filters have pairwise mirror-image symmetry in the frequency domain. In a paraunitary system, the analysis and synthesis filters are time-reversed versions of one another. Hence, both, the analysis and synthesis banks can be factored in terms of this structure. The filter banks realized using this factorization have the following properties:

- The filter bank is paraunitary, and therefore gives perfect reconstruction.

- The analysis and synthesis filters are time-reversed versions of each other. 
- The analysis and synthesis filters are all linear phase.

- The filters in the analysis and the synthesis banks both satisfy the pairwise mirrorimage property in the frequency domain.

It is interesting to note that the linear phase property along with the paraunitary condition implies that the analysis and synthesis banks are identical, up to a multiplier of \pm 1 on some of the filters, i.e., $F_{i}(z)= \pm H_{i}(z)$. This property is useful because, now, the same filter bank can be used for analysis and synthesis. This is useful in applications that involve encoding and decoding to be performed by the same system.

The variable matrices in our system are shown to be orthogonal matrices of a certain form. These orthogonal matrices can be characterised in terms of a fixed number of rotation angles. These rotation angles are actually the variables in the design process. Our factorization also gives a lattice structure for implementing these filter banks. If these angles are made the multipliers in the implementation, it gives rise to a structure that is robust to multiplier quantization. The properties of paraunitariness, linear phase, and pairwise mirror-image symmetry are retained no matter what the values of the angles are.

\section{References}

Crochiere R, Rabiner L 1983 Multirate digital signal processing. Signal Processing Series (Englewood Cliffs, NJ: Prentice Hall)

Mintzer F 1985 Filters for distortion-free two-band multirate filter banks. IEEE Trans. Acoust., Speech, Signal Process. 33: 626-630

Murnaghan 1962 The unitary and rotation groups (Washington, DC: Spartan)

Nguyen T, Vaidyanathan P P 1988 Maximally decimated perfect-reconstruction filter banks with pairwise mirror-image analysis (and synthesis) frequency responses. IEEE Trans. Acoust., Speech, Signal Process. 36: 693-706

Princen J, Bradley A 1986 Analysis/synthesis filter bank design based on time-domain aliasing cancellation. IEEE Trans. Acoust., Speech, Signal Process. 34: 1153-1161

Rioul O, Vetterli M 1991 Wavelets and signal processing. IEEE Signal Process. Mag. 8(Oct.): $14-38$

Smith M J T, Barnwell T P III 1984 A procedure for designing exact reconstruction filter banks for tree-structured sub-band coders. Proc. IEEE Int. Conf. Acoust., Speech, Signal Process., San Diego, pp 27.1.1-27.1.4

Soman A, Vaidyanathan P P 1992 Paraunitary filter banks and wavelet packets. Proc. Int. Conf. Acoust., Speech, Signal Process. pp 397-400

Soman A, Vaidyanathan P P 1993 Coding gain in paraunitary analysis synthesis systems. IEEE Trans. Signal Process. SP-41: 1824-1835

Soman A, Vaidyanathan P P, Nguyen T 1993 Linear phase paraunitary filter banks: theory, factorizations, and designs. IEEE Trans. Signal Process. (Special Issue on Wavelets) SP-41: 3480-3496

Vaidyanathan P P 1990 Multirate digital filters, filter banks, polyphase networks, and applications: A Tutorial. Proc. IEEE 78: 56-93

Vaidyanathan P P 1993 Multirate systems and filter banks. Signal Processing Series (Englewood Cliffs, NJ: Prentice Hall)

Vetterli M 1986 Filter banks allowing for perfect reconstruction. Signal Process. 10: 219-244

Vetterli M, Le Gall D 1989 Perfect reconstruction filter banks: some properties and factorizations. IEEE Trans. Acoust., Speech, Signal Process. 37: 1057-1071 